\title{
Mesure des doses absorbées neutron et gamma dans des fantômes soumis à une fluence mixte
}

\author{
E. BERAUD-SUDREAU, G. LEMAIRE, J. MAAS \\ (Manuscrit reçu le 23 janvier 1985)
}

\begin{abstract}
RÉSUMÉ
Les auteurs ont étudié, en premier lieu, les caractéristiques dosimétriques des jonctions PIN, puis ont comparé par des mesures dans l'air et en fantôme d'eau, dans un champ mixte neutron-gamma, les doses relevées par les jonctions PIN et le fluorure de lithium 7 aux doses obtenues à l'aide des techniques classiques de la dosimétrie en fluence mixte neutron-gamma.
\end{abstract}

La validité des réponses expérimentales de ces dosimètres étant établie, les auteurs évaluent la distribution des doses neutron et gamma dans des fantômes en plastique équivalent tissu (plastinaut) et animal (porcelet) ainsi que la répartition des doses au niveau des territoires médullaires de ce dernier.

Par ailleurs, un programme de calcul basé sur la méthode de Monte-Carlo a permis (à quelques hypothèses simplificatrices près) d'estimer les doses absorbées relatives au rayonnement primaire en provenance du réacteur SILENE à diverses profondeurs dans un fantôme d'eau cubique.

\section{ABSTRACT}

In order to study the dosimetric characteristics of PIN junctions, the absorbed doses measured by junctions and FLi7 in air and water phantoms were compared with the doses measured by classical neutron dosimetry in mixed fields.

The validity of the experimental responses of PIN junctions being thus checked and established, neutron and gamma dose distributions in tissue equivalent plastic phantoms (plastinaut) and mammals (piglets) were evaluated as well as the absorbed dose distributions in the pig bone-marrow producing areas.

By using correlatively a Monte-Carlo calculation method and applying some simplifying assumptions, the absorbed doses were derived from the spectrum of SILENE's neutrons at various depths inside a cubic water phantom and the results were compared with some from the literature.

\section{INTRODUCTION}

En radiobiologie, le paramètre physique de référence pour interpréter les résultats de l'irradiation de l'organisme est la dose absorbée au niveau des différents organes ou tissus. 
La seule connaissance de la réponse d'un dosimétre porté à la surface du corps ou placé dans l'air à l'endroit où se serait trouvé le centre du corps (midline tissue dose free in air) s'avère insuffisante car, au fur et à mesure que les rayonnements traversent l'organisme, la décroissance de leur fluence et les phénomènes de perte d'énergie du rayonnement ne sont pas négligeables : il s'établit un gradient de dose spécifique de la qualité (nature, énergie) du rayonnement. II importe donc de le déterminer car c'est la dose en profondeur effectivement reçue par les tissus ou organes considérés comme critiques qui permet de prédire la gravité d'une irradiation et de définir le traitement à entreprendre.

Par ailleurs, la relation entre dose absorbée et effet biologique doit encore être précisée, particulièrement en ce qui concerne l'efficacité biologique relative des rayonnements (EBR). II est maintenant établi que l'EBR des neutrons rapides par rapport aux rayonnements gamma s'accroît rapidement aux faibles doses et augmente quand l'énergie des neutrons diminue pour atteindre un maximum pour une énergie de $0,4 \mathrm{MeV}(2,5)$. II s'ensuit une variation de l'EBR quand la profondeur de pénétration dans l'organisme augmente.

L'objet de l'étude est de déterminer les doses neutronique et gamma déposées dans les différents territoires anatomiques d'un grand mammifère soumis, en flux monodirectionnel, à un champ mixte neutron-gamma.

A partir de ces doses, le radiobiologiste peut, ensuite, interpréter les effets observés au niveau de l'organisme entier et éventuellement proposer quelles doses retenir (dose à l'entrée, dose en tel ou tel point du corps, dose totale, dose propre à chaque rayonnement, etc.) en fonction du critère significatif auquel on prête attention.

\section{DEROULEMENT DE L'ETUDE}

L'étude comprend la mesure des doses neutron et gamma en différents points de l'organisme soumis à une fluence mixte neutron-gamma, après étalonnage des détecteurs, et la simulation de l'interaction de ces deux rayonnements avec la matière vivante pour comparer avec la mesure directe.

La partie théorique (simulation) consacrée au calcul des dépôts d'énergie par la méthode de Monte-Carlo est classique. Elle fait appel aux programmes Fortran du Centre d'analyse de défense de l'établissement technique central de l'armement (ETCA); elle est adaptée d'une part au champ du réacteur utilisé (réacteur Silène du Centre d'études nucléaires de Valduc), d'autre part aux deux fantômes dans lesquels les mesures sont faites : fantôme d'eau cubique de $60 \mathrm{~cm}$ d'arête et fantôme animal assimilé à un cylindre de $17,6 \mathrm{~cm}$ de diamètre pour le thorax et de $22 \mathrm{~cm}$ de diamètre pour l'abdomen.

La méthode théorique comporte des hypothèses simplificatrices qui conduisent à des incertitudes non appréciables directement. II faut donc confronter ses résultats avec ceux obtenus expérimentalement. Cette comparaison a été effectuée, pour le fantôme cubique rempli d'eau, à l'aide de chambres d'ionisation pour mesurer les doses en profondeur; 
elle a permis de déterminer le degré de confiance à accorder au calcul développé pour le fantôme animal cylindrique. Elle était également nécessaire à l'étude des réponses spectrales du système dosimétrique retenu pour les mesures en fantômes solides (plastinaut et animal congelé), pour lesquels les chambres d'ionisation ne sont plus adaptées.

Les mesures à l'intérieur des fantômes ont été complétées sur l'animal vivant par celles des doses reçues par le système dosimétrique précédent, mais placé sur chaque flanc, et par celles de l'activation du soufre 32 des soies des flancs proximal et distal par rapport à la source de rayonnements mixtes neutron-gamma.

\subsection{Conditions d'irradiation auprès du réacteur Silène}

Silène est un réacteur homogène expérimental utilisant une solution fissile de nitrate d'uranyle enrichie à $93 \%$ en uranium 235 et contenue dans une cuve annulaire en acier de $35 \mathrm{~cm}$ de diamètre traversée en son axe par un canal de $7 \mathrm{~cm}$ de diamètre où se déplace la barre de contrôle. La solution est introduite dans la cuve jusqu'à un niveau surcritique prédéterminé et l'excursion de puissance est provoquée par l'extraction de la barre. En fonction de la nature de la barre et de sa vitesse d'éjection, trois types de fonctionnement peuvent être obtenus : en salve, en libre évolution ou en palier.

Le flanc gauche des fantômes animaux (porcelets congelés) ou les faces avant des autres fantômes ont été placés à $3 \mathrm{~m}$ du réacteur sauf indication contraire. A cette distance et compte tenu des dimensions de la cellule d'irradiation $(12 \times 19 \mathrm{~m})$, le rayonnement diffusé par les parois et le sol n'est pas négligeable par rapport au rayonnement direct.

Le spectre neutronique à $3 \mathrm{~m}$ du réacteur est représenté sur la figure 1 et les fluences neutroniques pour $10^{17}$ fissions sont :
Fluence thermique
Fluence intermédiaire
Fluence rapide
(0 à $0,4 \mathrm{eV})$
$(0,4 \mathrm{eV}$ à $0,5 \mathrm{MeV})$
$(0,5 \mathrm{MeV}$ à $10 \mathrm{MeV})$

: $3,8 \cdot 10^{10}$ n.cm $\mathrm{cm}^{2}$

$: 8,8 \cdot 10^{10}$ n. $\mathrm{cm}^{-2}$

$: 7,0.10^{10}$ n. $\mathrm{cm}^{-2}$

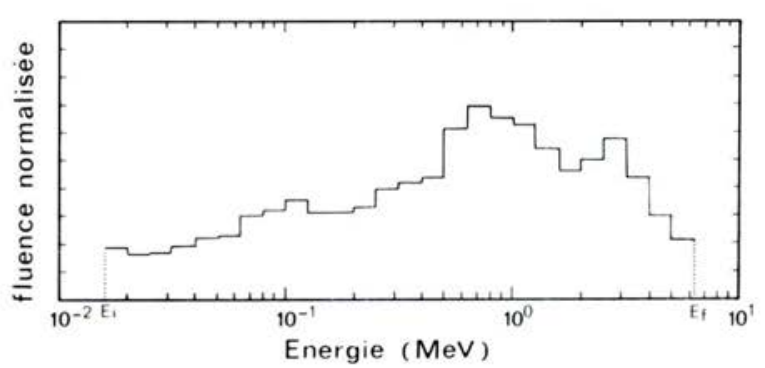

VOL. $20 \cdot \mathrm{N}^{\circ} 2$
Fig. 1. - Distribution énergétique des neutrons du réacteur Silène. (Spectrométrie par protons de recul) [BENEZECH communication personnelle, 1975]. 
A cette distance de la source fissile, le rapport des doses absorbées gamma et neutron est voisin de 1,2.

\subsection{Techniques dosimétriques - Etalonnage des détecteurs}

En champ mixte, la détermination des composantes neutron et gamma de la dose absorbée nécessite l'emploi de deux dosimètres présentant des sensibilités différentes aux deux types de rayonnements. Généralement, l'un des dosimètres ( $T$ ) possède approximativement une sensibilité équivalente aux neutrons et aux photons, alors que l'autre (U) est surtout sensible aux photons. Les quotients, $R_{T}$ et $R_{Y}^{\prime}$ des réponses des dosimètres à leur sensibilité aux photons gamma de référence sont alors donnés (ICRU, 1977) par les deux équations suivantes (6) :

$$
R_{T}^{\prime}=k_{T} D_{N}+h_{T} D_{G} \text { et } R_{U}^{\prime}=k_{U} D_{N}+h_{U} D_{G}
$$

où: $D_{N}, D_{G}$ : dose absorbée due respectivement aux neutrons et aux photons;

$k_{T}, k_{U} \quad$ : rapport de la réponse de chaque dosimètre aux neutrons à sa réponse aux photons de référence ;

$h_{T}, h_{U} \quad$ : rapport de la réponse de chaque dosimètre aux photons à sa réponse aux photons de référence.

En général, l'utilisation d'un couple de chambres d'ionisation apparaît comme la méthode la mieux adaptée aux mesures des doses absorbées neutron et gamma car leur relative miniaturisation, leur souplesse d'emploi, leur sensibilité, leur fidélité et leur précision en font des détecteurs de choix. Mais, pour des mesures à débit de dose élevé ou des mesures en fantôme solide, il faut recourir à un autre système dosimètrique, la plupart du temps constitué par un couple de détecteurs dont les sensibilités et les caractéristiques sont plus ou moins bien connues.

Pour réaliser des mesures ponctuelles ou quasi ponctuelles dans le plastinaut et le fantôme animal, le choix s'est porté sur une jonction PIN comme dosimètre neutron et sur le fluorure de lithium 7 comme dosimètre gamma.

La dosimétrie de référence a été réalisée à l'aide du système des doubles chambres d'ionisation selon la méthode préconisée par Rossi et effectuée en collaboration avec le laboratoire de biophysique appliquée du département de protection au Commissariat à l'énergie atomique (CEA). Une chambre est appelée "chambre ET" car ses parois sont en plastique équivalent aux tissus de Shonka (gaz de remplissage équivalent aux tissus de Rossi) et l'autre "chambre Alu" car elle est construite en aluminium (gaz de remplissage : argon). La chambre "ET" mesure la dose totale (neutron + gamma) alors que la chambre "Alu" ne mesure que la composante gamma de la fluence mixte.

\subsubsection{Jonction PIN}

C'est une diode au silicium dont les modifications des caractéristiques électriques permettent de mesurer la dose absorbée après irradiation neutronique (création de défauts au niveau de la structure cristalline). 
La méthode de lecture retenue a été la mesure de la chute de tension après irradiation neutronique, car bien que le problème de lecture en temps de recouvrement ait été résolu, les diodes dosimètres fournies par les constructeurs présentent une dispersion trop importante de leur temps de recouvrement initial qui aurait obligé à un repérage préalable de chacune des diodes ou à une utilisation par lots après tri. Le dispositif de lecture est commercialisé par Studsvik.

La réponse de la jonction PIN en fluence neutronique est donnée (fig. 2) en fonction de la dose absorbée. La chute de tension directe $=f($ dose) n'est pas linéaire sur l'ensemble de l'intervalle de dose. La courbe d'étalonnage devient en effet curviligne aux doses les plus faibles analysées. Elle a été établie par comparaison dans l'air avec la dose due aux neutrons, déterminée par la technique classique des deux chambres (équivalent tissu et aluminium) auprès du réacteur Silène (à $3 \mathrm{~m}$ ) pour des doses de 0,4 Gy à 15 Gy.

Fig. 2. - Courbe d'étalonnage des jonctions auprès du réacteur Silène en terme de dose absorbée (lecteur Studsvik type 3809 A).

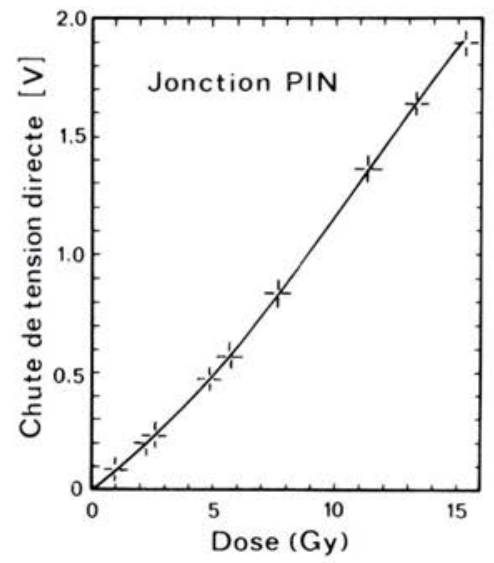

\subsubsection{Fluorure de lithium 7}

C'est un halogénure alcalin dont la thermoluminescence est utilisée pour mesurer la dose absorbée après son irradiation. Le dosimètre commercialisé par la société Harshaw est le TLD-700. Utilisé une seule fois, il ne subit aucun traitement de régénération. Ce dosimètre est pratiquement insensible aux neutrons et fournit donc, en fluence mixte, la dose gamma totale.

L'intégration des pics de thermoluminescence représente l'énergie lumineuse libérée qui est proportionnelle à la dose absorbée dans le composé. Le lecteur comprend un système de chauffage et un ensemble photomultiplicateur. Le courant issu de ce dernier est emmagasiné dans une capacité dont la charge représente la dose absorbée.

La figure 3 représente la courbe d'étalonnage obtenue avec les rayonnements gamma de référence, c'est-à-dire les rayonnements gamma émis par le cobalt 60 . 


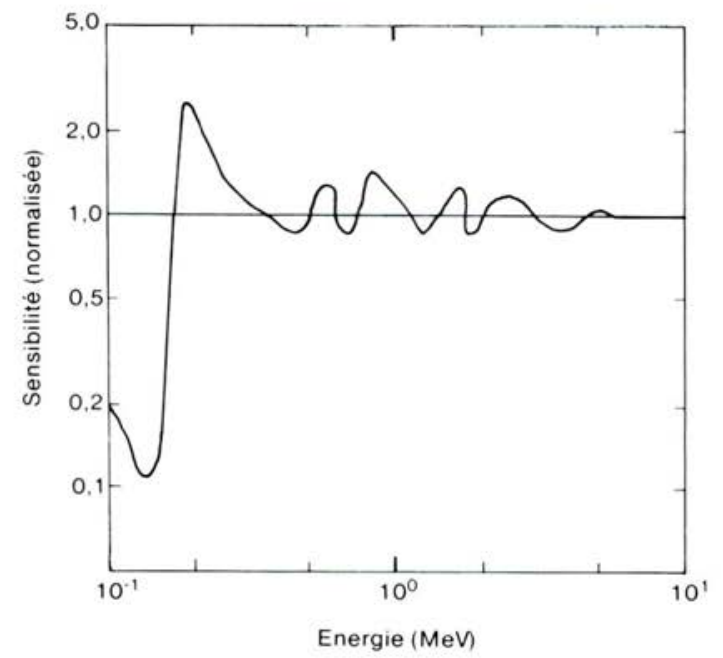

Fig. 2 bis. - Sensibilité théorique de la diode au silicium aux neutrons, normalisée par unité de kerma tissu muscle ICRU.

\subsection{Dosimétrie en fantômes}

Avant d'implanter les dosimètres dans le plastinaut et le fantôme animal, il fallait vérifier qu'à l'intérieur des fantômes leur réponse demeurait la même malgré la dégradation du spectre neutronique. En effet, le point important est que la diode dosimètre est peu sensible aux neutrons d'énergie inférieure à $0,2 \mathrm{MeV}$.

Différentes expériences ont confirmé qu'elle était totalement insensible aux neutrons thermiques (fig. 2 bis). Etant donné la réponse en énergie de la diode, il est alors apparu nécessaire d'effectuer une dosimétrie comparative diodes-chambres en fantôme liquide. Ceci a été fait en fantôme d'eau.

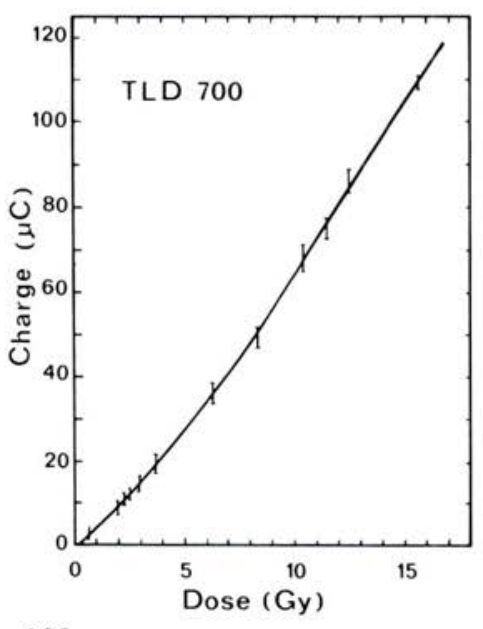

122
Fig. 3. - Etalonnage du TLD700 en terme de dose absorbée (Lecteur Harshaw type 2000 A). Fluence gamma du ${ }^{60} \mathrm{Co}$. 


\subsubsection{Fantôme d'eau}

C'est une cuve cubique de $60 \mathrm{~cm}$ d'arête à parois en plexiglas $(1,5 \mathrm{~cm}$ d'épaisseur). En raison des problèmes pratiques posés par l'utilisation d'un liquide équivalent aux tissus et selon les recommandations de l'ICRU [6], le liquide est l'eau.

Les résultats sont retranscrits sur la figure 4 pour le dosimètre neutron (jonction PIN) auprès du réacteur Silène et sur les figures 5 et 6 pour le dosimètre gamma (TLD-700) auprès respectivement d'une source de cobalt 60 et du réacteur Silène.

Fig. 4. - Comparaison des doses neutron mesurées par chambres ET et ALU et jonctions PIN à $3 \mathrm{~m}$ du réacteur (tir en palier) dans le fantôme d'eau avec les doses calculées par Monte-Carlo, en tenant compte de la non-réponse de la diode en dessous de 0,2 MeV mais sans prendre en compte le diffusé par la salle. La courbe hachurée correspond à l'extrapolation de la figure 6.

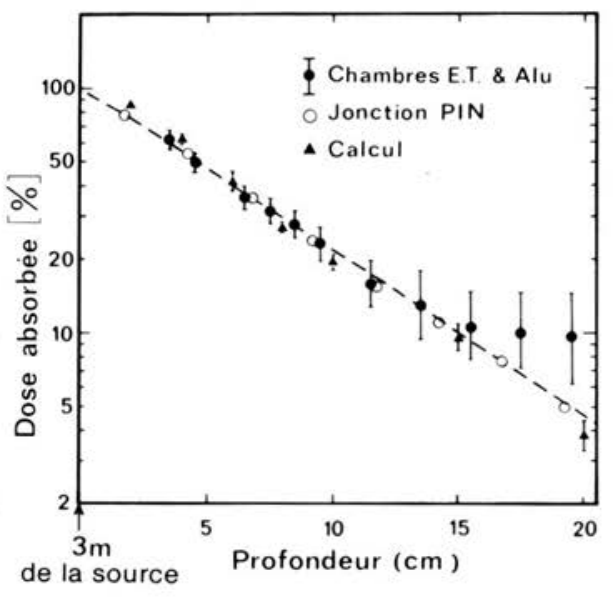

La figure 4 montre que l'accord entre les réponses des chambres et celles des jonctions est excellent jusqu'à une profondeur de $15 \mathrm{~cm}$. Au-delà, la méthode des doubles chambres fournit pour la dose neutron des valeurs plus élevées, affectées d'erreurs plus importantes compte tenu du mode de détermination. Cette sous-réponse des diodes peut être expliquée par les caractéristiques intrinsèques de la diode qui ne répond plus aux neutrons d'énergie inférieure à $0,2 \mathrm{MeV}$. En effet, quand on s'enfonce dans le fantôme d'eau, le spectre se dégrade et le rayonnement diffusé provenant de l'arrière de la salle où est implanté le réacteur n'est plus négligeable. Dans ces conditions, il peut paraître normal d'obtenir une sous-réponse des diodes.

Les figures 5 et 6 montrent un excellent accord entre les réponses des chambres et celles du TLD-700 à toutes les profondeurs pour la dose gamma en fluence gamma pure $\left({ }^{60} \mathrm{Co}\right)$ comme en fluence neutron-gamma (Silène), ce qui ne permet pas de conclure quant à la nature de la divergence précédente. Par ailleurs, la comparaison des doses neutron mesurées et calculées pour le fantôme d'eau jusqu'à $15 \mathrm{~cm}$ (fig. 4) montre que la corrélation peut être considérée comme excellente car, avec ce fantôme, géométrie, composition, densité, sections efficaces et kerma sont bien connus, contrairement au fantôme tissu. D'autre part, le calcul prend en compte la non réponse de la diode en dessous de 0,2 MeV. 


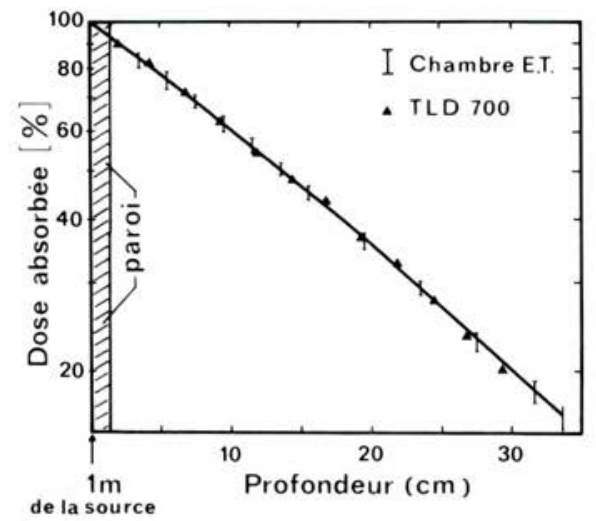

Fig. 5. - Comparaison des doses gamma mesurées par chambre ET et TLD 700 dans le fantôme d'eau $(60 \times 60 \times 60 \mathrm{~cm})$ placé à $1 \mathrm{~m}$ d'une source de ${ }^{60} \mathrm{Co}$ non collimatée (incertitude totale chambre ET voisine de $3 \%$ ).

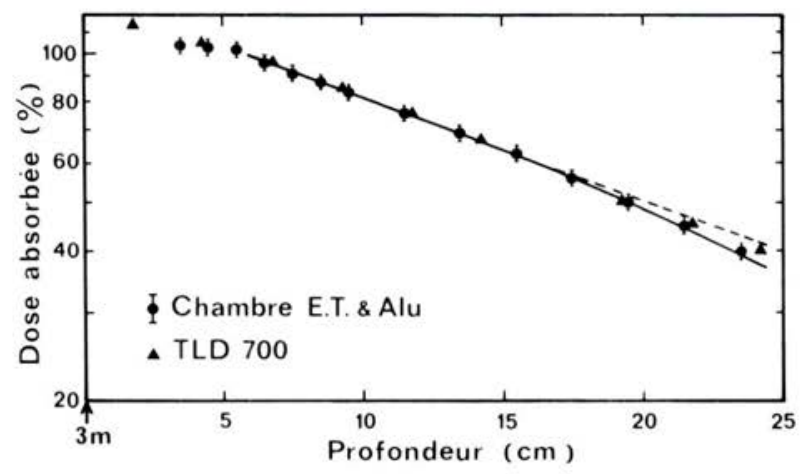

Fig. 6. - Comparaison des doses gamma (chambres ET et ALU et TLD 700) mesurées auprès du réacteur Silène. La droite hachurée permet de calculer les doses neutron de la figure 4.

La figure 7 montre les gradients de dose neutron et gamma qui s'établissent au sein du fantôme d'eau. Les mesures n'ont pas été réalisées au-delà de $20 \mathrm{~cm}$, compte tenu de l'épaisseur du fantôme animal et de l'objet réel de l'étude.

\subsubsection{Plastinaut}

Ce fantôme permet de simuler le corps humain. II mesure $1,70 \mathrm{~m}$, pèse $70 \mathrm{~kg}$ et est composé d'un plastique équivalent aux tissus humains vis-à-vis des neutrons et des photons, avec squelette réel incorporé et artifice de polymérisation du plastique pour simuler les poumons. 


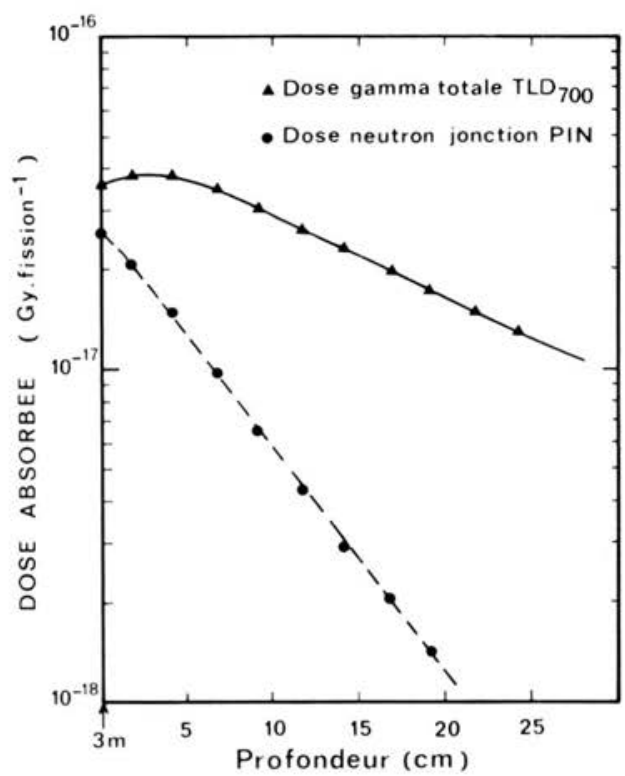

Fig. 7.- Distributions des doses absorbées neutron et gamma mesurées dans le fantôme d'eau (tir en salve).
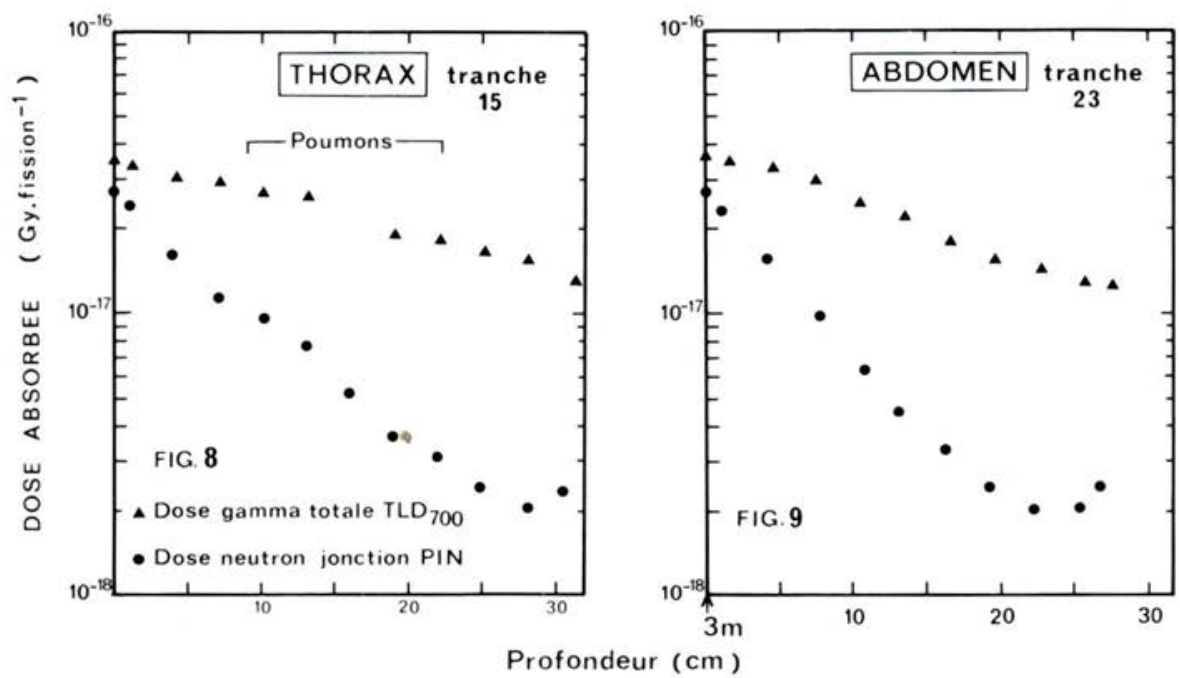

Fig. 8 et 9. - Distributions des doses absorbées neutron et gamma mesurées au sein d'un fantôme anthropomorphe (plastinaut) lors d'un tir en libre évolution.

Les figures 8 et 9 montrent, en fonction de l'épaisseur, la variation des doses neutron et gamma au sein du plastinaut. Elles mettent nettement 
en évidence un pouvoir d'arrêt moins important des rayonnements au niveau du thorax dû à la faible densité des tissus pulmonaires et, dans les derniers centimètres, la remontée des doses neutrons due à l'influence du rayonnement diffusé atteignant le fantôme par l'arrière, ces neutrons étant pour beaucoup rapides comme le montrent les mesures d'activation du soufre des soies distales.

Les doses neutrons mesurées ont été confrontées aux valeurs que nous pouvons calculer pour le fantôme d'Auxier [1] et à celles calculées pour le fantôme de Snyder [8] par Dousset [4] lors des divergences Crac, le spectre de Crac étant voisin de celui de Silène. Les fantômes d'Auxier et de Snyder ont la même géométrie et la même dimension (cylindre tissu de $30 \mathrm{~cm}$ de diamètre et de $60 \mathrm{~cm}$ de haut), mais diffèrent dans leur composition et le découpage des éléments de volume dans lesquels les doses sont calculées.

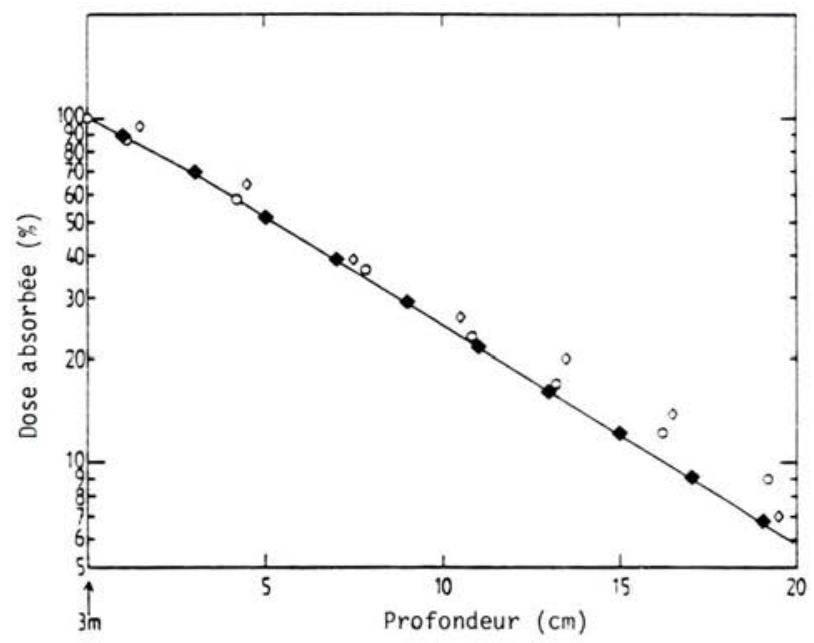

- Plastinaut - tranche 23 (Silène)

- Fantôme d'Auxier - tranche 3 (Silène)

- Fantôme de Snyder - tranche centrale (Crac)

Fig. 10. - Comparaison de la distribution des doses absorbées neutron dans divers fantômes équivalents aux tissus.

La figure 10 montre les résultats de cette confrontation. On observe une concordance satisfaisante pour les dix premiers centimètres et une dispersion au-delà (l'influence du rayonnement diffusé n'est pas prise en compte dans les calculs). On note également l'atténuation monotone pour le fantôme de Snyder contrairement au fantôme d'Auxier, ce qui peut s'expliquer pour le premier par de meilleurs facteurs de conversion fluencedose, un nombre de tirages aléatoires effectués plus élevé et des éléments de volume plus petits. 


\subsubsection{Fantôme animal}

Les résultats précédents montrent que les TLD-700 associés aux jonctions PIN constituent un système dosimétrique adapté aux mesures des doses neutrons et gamma en fantôme soumis à une fluence mixte.

Pour recouper ces résultats, les distributions des doses neutron et gamma ont été mesurées au niveau du thorax et de l'abdomen d'un fantôme animal (porcelet congelé placé à $3 \mathrm{~m}$ du réacteur, flanc gauche en face proximale). Des conteneurs en plexiglas renfermant 3 TLD-700 et 3 jonctions PIN ont été implantés le long de deux axes passant par le thorax et l'abdomen (imprécisions du positionnement : $\pm 5 \mathrm{~mm}$ ).

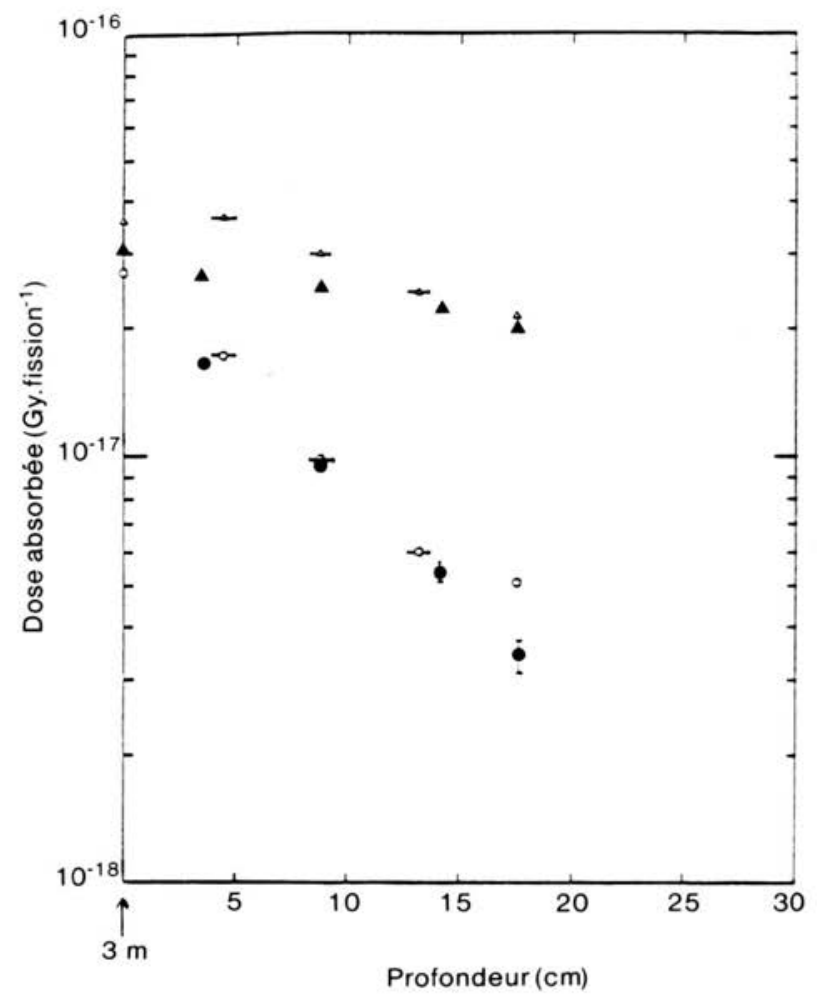

- Dose gamma totale (TLD-700)

- Dose neutron (Jonction PIN)

- Dose gamma primaire (calcul)

- Dose neutron (calcul)

Fig. 11. - Distribution des doses gamma totale (TLD-700), gamma primaire (calcul) et neutron en profondeur au niveau du thorax du fantôme animal

(tir en libre évolution). 


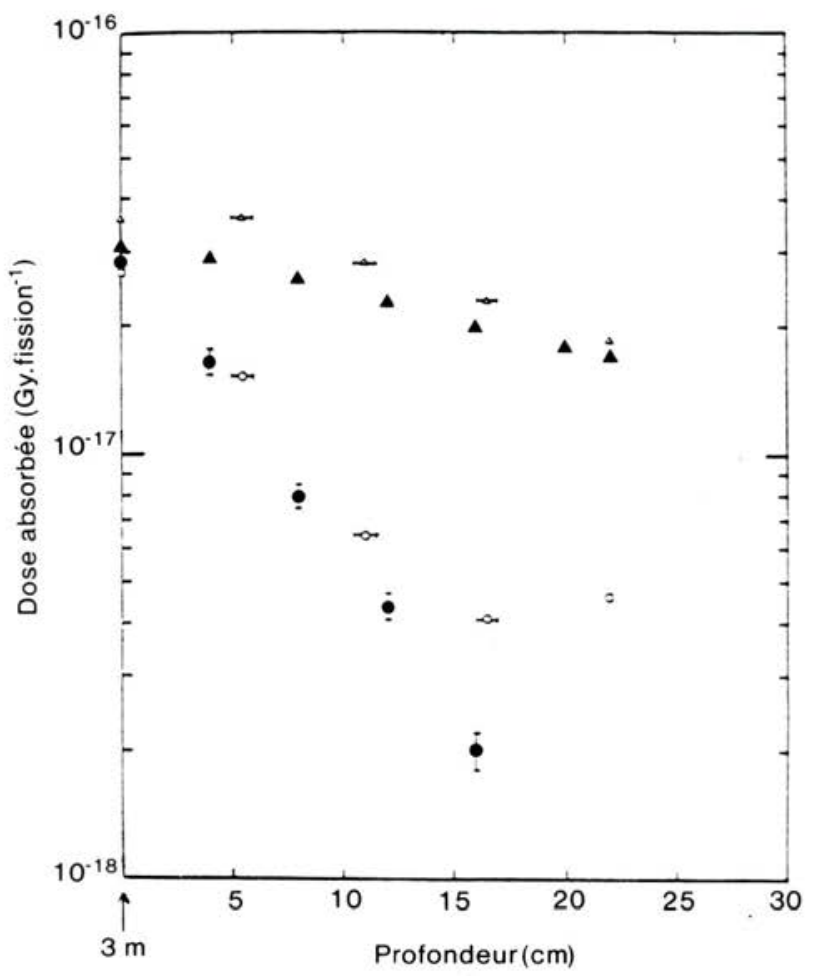

- Dose gamma totale (TLD-700)

- Dose neutron (Jonction PIN)

- Dose gamma primaire (calcul)

- Dose neutron (calcul)

Fig. 12. - Distribution des doses gamma totale (TLD-700), gamma primaire (calcul) et neutron en profondeur au niveau de l'abdomen du fantôme animal (tir en libre évolution).

Les figures 11 et 12 montrent des résultats comparables à ceux du plastinaut avec, cependant, un effet de densité du tissu pulmonaire moins important et, pour le calcul Monte-Carlo, un gradient de dose plus prononcé qu'avec les mesures. Compte tenu des approximations faites et de la non prise en compte du rayonnement diffusé, on peut estimer que les résultats calculés sont corrects.

En utilisant l'étude d'Auxier, faite lors des expériences de Brown [3] sur un porc de $150 \mathrm{~kg}$ placé à $3 \mathrm{~m}$ (midline) du réacteur HPRR, pour l'adapter à la distance de $3 \mathrm{~m}$ face avant, on obtient la comparaison de la figure 13. On remarque, dans l'expérience de Brown, l'influence du rayonnement diffusé à partir de $25 \mathrm{~cm}$ d'épaisseur et la plus grande atténuation de la dose neutron qui peut s'expliquer par la différence des spectres des deux réacteurs et la différence des poids des animaux. 


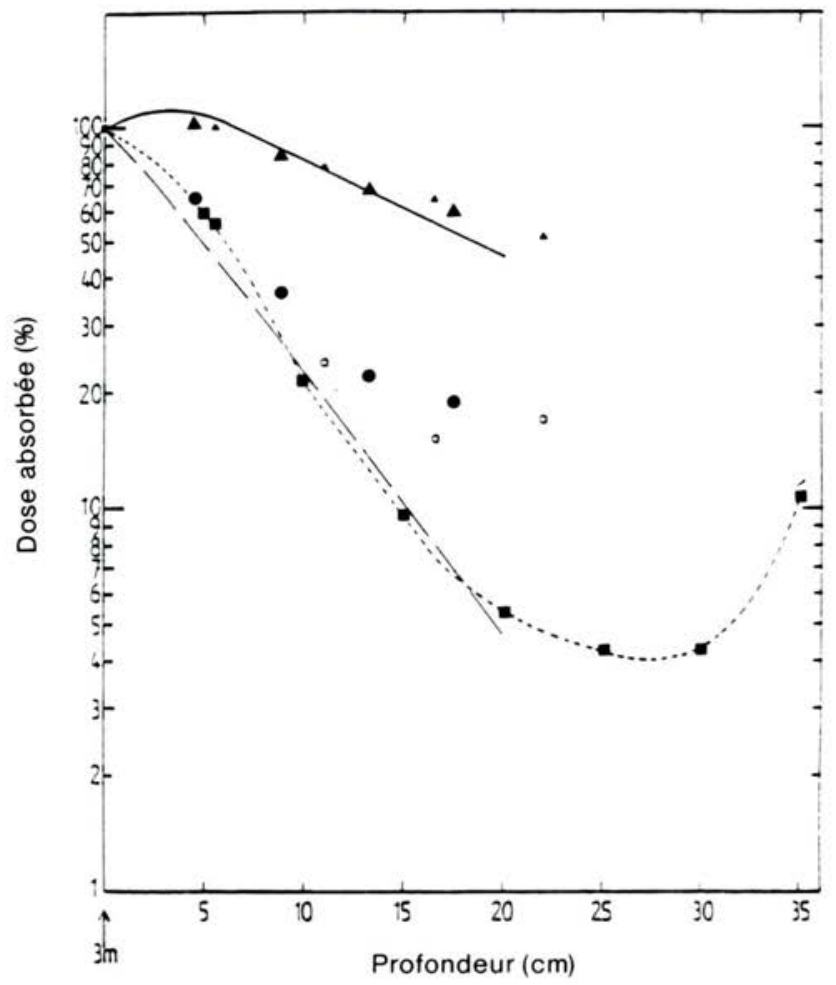

Dose gamma totale (TLD-700)

- Fantôme d'eau

- Thorax fantôme animal

- Abdomen fantôme animal

Dose neutron (Jonction PIN)

-- Fantôme d'eau

- Thorax fantôme animal

- Abdomen fantôme animal

$\rightarrow$ Dose neutron exp. Brown (HPRR)

Fig. 13. - Comparaison des distributions des doses absorbées neutron et gamma dans divers fantômes.

A Silène, le rapport de la dose maximale à la dose minimale est proche de 7 pour les neutrons et de 2 pour les photons sans tenir compte de la loi en $1 / d^{2}$, ce qui représente des conditions d'irradiation "non uniformes" d'après les recommandations de l'ICRU [7], et explique la nécessité de préciser les doses reçues par les organes ou tissus en cause dans l'effet observé après irradiation de l'organisme. Pour les répercussions héma- 


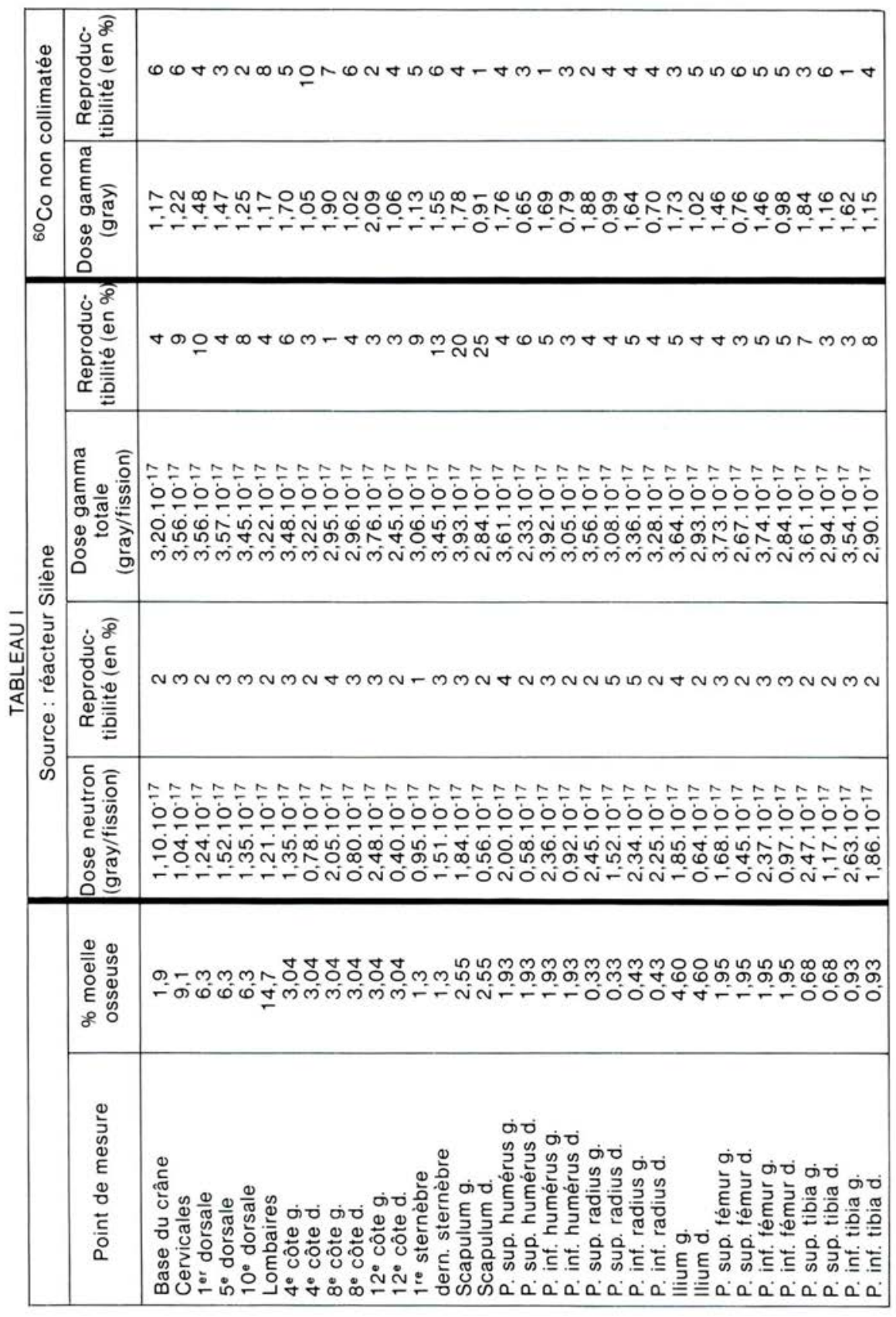


tologiques et la survie à 30 jours, il s'agit du tissu hématopoïétique. C'est pourquoi des implants osseux (trois TLD-700 et trois jonctions PIN) ont été effectués dans de nombreux territoires médullaires d'un fantôme animal placé à $3 \mathrm{~m}$ (midline) comme pour les expérimentations biologiques.

Le tableau I récapitule les résultats et montre d'une part l'excellente reproductibilité des mesures, d'autre part l'influence du facteur géométrie sur la dose au niveau d'un membre, selon que ce dernier est ou non masqué au rayonnement incident.

Par ailleurs, les doses externes sur les flancs et sur les lignes dorsale et ventrale du fantôme animal ont été mesurées à l'aide du même système dosimétrique (tableau II).

TABLEAU ॥

\begin{tabular}{|l|c|c|}
\hline \multirow{2}{*}{ Flanc gauche } & $\begin{array}{c}\text { Dose gamma totale } \\
\text { tissue dose free in air (Gy) }\end{array}$ & $\begin{array}{c}\text { Dose neutron } \\
\text { Flanc droit }\end{array}$ \\
\cline { 2 - 3 } Ligne sagittale (dos) & $3,9 \pm 0,1$ & $2,8 \pm 0,1$ \\
Ligne sagittale (abdomen) & $2,2 \pm 0,1$ & $0,55 \pm 0,1$ \\
& 3,3 & 2,1 \\
\hline
\end{tabular}

L'atténuation proximale-distale est donc de 1,8 pour les photons et de 5,1 pour les neutrons.

Les mesures externes dorsales donnent une dose neutron plus faible que dans l'air car le fantôme animal fait écran aux neutrons diffusés par le sol (2,1 contre 2,5 Gy) et une dose gamma plus forte (3,3 contre 2,95 Gy), ce renforcement provenant du rayonnement diffusé par les tissus et des réactions $(n, \gamma)$. Dans le cas des mesures externes ventrales, le renforcement des doses provient de la diffusion des rayonnements par le sol, surtout pour les photons.

\subsection{Mesures sur l'animal vivant}

En mesurant l'activation du soufre 32 des soies prélevées sur les flancs, il est possible d'apprécier expérimentalement le rapport des doses entrée/sortie des rayonnements dans les conditions exactes de l'irradiation. Le phosphore 32 est induit par réaction nucléaire sur le soufre 32 , ${ }^{32} \mathrm{~S}(\mathrm{n}, \mathrm{p}){ }^{32} \mathrm{P}$, dont le seuil se situe aux environs de $2 \mathrm{MeV}$ et dont la section efficace devient constante et égale à 300 millibarns dès que l'énergie des neutrons atteint $4 \mathrm{MeV}$. Les soies sont prélevées sur les flancs et leur activité en phosphore 32 est mesurée soit par comptage bêta direct soit par scintillation liquide.

Le tableau III présente les résultats pour une "midline tissue dose neutron free in air", à $3 \mathrm{~m}$ du réacteur, comprise entre 3,05 et 4,7 Gy. II montre une variabilité certaine, attribuable partiellement aux différences d'épaisseur des animaux et surtout aux imprécisions de la mesure de l'activité des soies du flanc droit car, du fait de l'atténuation dans le corps, 
cette activité est voisine du bruit de fond de l'appareillage de détection : le coefficient de variation de la mesure d'activité d'un même échantillon passe de $10 \%$ pour le flanc gauche à plus de $30 \%$ pour le flanc droit. Pour la dose de 4,6 - 4,7 Gy la valeur moyenne du rapport flanc gauche/flanc droit est de 4,6 ce qui est peu différent de la valeur fournie par les dosimètres placés à la surface du fantôme animal $(5,1)$.

TABLEAU III

Rapport flanc gauche/flanc droit de l'activité en ${ }^{32} \mathrm{P}$ des soies du porc

(Réacteur Silène sans écran)

\begin{tabular}{|c|c|c|c|c|c|}
\hline $\begin{array}{l}\text { Nombre } \\
\text { d'animaux }\end{array}$ & $\begin{array}{c}\text { Midline tissue dose free in air } \\
\text { à } 3 \text { m (Gy) }\end{array}$ & \multicolumn{4}{|c|}{ Rapport $\frac{\text { flanc gauche }}{\text { flanc droit }}$} \\
\hline 2 & 3,05 & \multicolumn{4}{|c|}{$\begin{array}{l}1,7 \\
3,5\end{array}$} \\
\hline 1 & 3,25 & \multicolumn{4}{|c|}{6,0} \\
\hline 1 & 3,40 & \multicolumn{4}{|c|}{4,9} \\
\hline 11 & $4,6 \cdot 4,7$ & $\begin{array}{l}4,9 \\
4,0 \\
4,2\end{array}$ & $\begin{array}{l}5,7 \\
4,1 \\
4,9\end{array}$ & $\begin{array}{l}4,2 \\
3,8 \\
4,4\end{array}$ & $\begin{array}{l}4,3 \\
5,9\end{array}$ \\
\hline
\end{tabular}

Cette méthode n'est malheureusement qu'une mesure des neutrons rapides dont l'énergie est au moins supérieure à $2 \mathrm{MeV}$. Elle ne donne bien évidemment aucun renseignement sur les neutrons d'énergie inférieure.

\section{CONCLUSION}

Au plan du calcul théorique des doses absorbées neutron et gamma au sein de fantômes, cette étude a permis l'adaptation des programmes du Centre d'analyse de défense de l'ETCA au flux mixte du réacteur Silène en configuration nue et aux fantômes dans lesquels les mesures sont faites. Cette adaptation conduit à montrer que les différences constatées entre valeurs théoriques et valeurs expérimentales pourraient être réduites en partie grâce à une meilleure connaissance des spectres neutronique et gamma du réacteur et s'expliquent, pour le reste, par les imprécisions affectant certaines données d'entrée et dues, notamment, aux simplifications introduites par la modélisation, à la moins bonne connaissance des sections efficaces et des facteurs Kerma de certains éléments, à la non prise en compte, pour le calcul, des phénomènes de diffusion par le sol et les murs.

Les résultats du calcul peuvent être améliorés par une meilleure connaissance des spectres neutrons et gamma émis. Ce travail est actuellement entrepris conjointement par une équipe du département de protection sanitaire du CEA et une équipe du département détection protection nucléaire de l'ETCA.

Au plan expérimental, l'étude montre que le système dosimétrique TLD-700 - jonction PIN est bien adapté aux mesures quasi ponctuelles 
des doses absorbées en fantômes soumis à un champ mixte neutrongamma. Le protocole de lecture des doses a été défini. L'essentiel des incertitudes de mesure absolue de la dose tient plus aux problèmes d'étaIonnage qu'aux réponses spectrales. La surréponse de la chambre "Alu" en profondeur dans le fantôme d'eau a été mise en évidence. En fantôme animal, la reproductibilité des mesures s'est avérée excellente ; en irradiation unilatérale, les distributions des doses absorbées neutrons et gamma ont été déterminées de manière très détaillée. On peut donc considérer, à l'heure actuelle, la jonction PIN comme un excellent dosimètre de criticité et l'étude complète de ses caractéristiques doit être poursuivie.

Les distributions de dose calculées et mesurées obtenues sont en accord avec celles d'autres auteurs en géométrie et champ similaires. Le gradient de dose neutron global peut être évalué à l'aide de ce système dosimétrique disposé sur les faces d'entrée et sortie. II peut l'être aussi du moins pour la partie du spectre neutronique d'énergie supérieure à $2 \mathrm{MeV}$ - en mesurant l'activation du ${ }^{32} \mathrm{~S}$ des soies du porc. Remarquons, toutefois, que cette méthode est rudimentaire et ne peut donner qu'une approximation grossière.

En irradiation unilatérale, la forte hétérogénéité des doses absorbées, mise en évidence dans un fantôme animal de dimensions voisines de celles de l'homme, montre la nécessité de connaître cette distribution, car c'est la dose effectivement reçue par les tissus ou organes en cause dans l'effet observé qui conditionne cet effet. La connaissance des doses à l'entrée comme à la sortie et celle de la midline tissue dose pourraient conduire à préciser laquelle de ces doses traduirait le mieux les conditions de l'irradiation et son pronostic. Les expériences ultérieures sur des animaux comporteront la mesure de ces doses à l'aide de ce système dosimétrique. pour les différents champs mixtes neutron-gamma utilisés.

\section{REFÉRENCES}

[1] AUXIER J.A., SNYDER W.S. et JONES T.D. Neutron interactions and penetration in tissue. In : Radiation dosimetry (Attix F.H., Roesch W.C. and Tochilin E., Eds.). New York: Academic Press, 1968, vol. I, 275-316.

[2] BROERSE J.J., BARENDSEN G.W. Relative biological effectiveness of fast neutrons for effects on normal tissues. Curr. Top. Radiat. Res. 1973, 8, 305-309.

[3] BROWN D.G., JOHNSON D.F., AUXIER J.A. Unilateral and bilateral exposure of swine to fission neutrons. Health Phys., 1971, 21, 537-545.

[4] DOUSSET M., PARMENTIER N., SOUDAIN G., BRICKA M. Dose et transfert d'énergie linéique à l'intérieur de l'organisme humain exposé au rayonnement mixte neutron-gamma de la source CRAC. In : 1. Symposium on neutron dosimetry in biology and medicine, Neuherberg/München, 15-19 May 1972 (Burger G., Schraube H., Ebert H.G., Eds). (EUR-4896). Luxembourg: Commission of the European Communities, 1972, vol. 2., 673-700.

[5] HALL E.J. RBE and OER values as a function of neutron energy. Europe J. Cancer, $1974,10,297$.

[6] INTERNATIONAL COMMISSION ON RADIATION UNITS AND MEASUREMENTS (ICRU). Neutron dosimetry for biology and medicine, ICRU Report 26. Washington : ICRU, 1977. 


\section{E. BERAUD-SUDREAU, G. LEMAIRE, J. MAAS}

[7] INTERNATIONAL COMMISSION ON RADIATION UNITS AND MEASUREMENTS (ICRU). Radiation quantities and units, ICRU Report 33. Washington : ICRU, 1980.

[8] NATIONAL COUNCIL ON RADIATION PROTECTION AND MEASUREMENTS (NCRP). Protection against neutron radiation, NCRP Report 38. Bethesda : NCRP, 1971. 